\title{
Method of the Year 2018: Imaging in freely behaving animals
}

\author{
Neuronal imaging in unrestrained animals has expanded the range of behaviors amenable to circuit-level studies in \\ several model organisms.
}

A dvances in imaging technology and in fluorescent sensors have allowed detailed analyses of the neuronal activity underlying a variety of behaviors in animals as diverse as rats, mice, fish, flies and worms. Restraining animals gives experimenters exquisite control over the sensory environment of their subjects, which can be advantageous for studies of visually guided or olfactory-guided behaviors, for example. However, restrained animals are limited in their ability to exhibit more complex behaviors, particularly social behaviors. Technological developments in recent years have made it possible to image neuronal activity in freely behaving animals. For its potential to allow scientists to study the neuronal basis of naturalistic behaviors at the cell, circuit and whole-brain levels, we have chosen imaging in freely behaving animals as Method of the Year 2018.

Miniature microscopes have left mice and rats free to go about their naturalistic behaviors for almost two decades. Although the first miniature microscopes were rather heavy and could be used only with rats, later versions were further miniaturized, and these tools can now be applied in mice without overly adverse effects on animal behavior. Recent developments have further expanded the imaging modalities that can be used with freely behaving rodents. In addition to wide-field implementations, miniature microscopes with two-photon or volumetric imaging capabilities are now available. A Comment by Aharoni et al. discusses the miniature-microscope options accessible to researchers and mentions further developments that are on the horizon for these microscopes.

Miniature microscopes are now being used in an increasing number of labs to study behaviors as diverse as spatial navigation, sleep and social interactions. Their application is even being explored in marmosets. These microscopes can also help to visualize aberrant brain activity in neuropathological conditions in animal models. A News Feature surveys how researchers monitor activity in the brain with miniature microscopes, demonstrating the impact this technology has had and continues to have in systems neuroscience.

While the benefits of imaging neuronal activity with miniature microscopes are obvious in the context of social or predatory behaviors, this technology may also provide additional mechanistic insights for behaviors that are traditionally studied in restrained animals. For instance, miniature microscopy technology avoids confounds introduced by rigid head restraint. And although there is a trade-off in terms of experimental control over the environment, the animals can make use of additional sensory input, such as, for example, from the vestibular system. Such considerations are brought up in a Comment by Wallace and Kerr.

Miniature microscopes have become invaluable for many rodent behavioral studies, but this technology is not compatible with behavioral studies in smaller animals such as fish, flies and worms. A feasible alternative for monitoring brain activity in these animals involves tracking the animals while they move freely in a behavioral arena. The positional information acquired through tracking can then be used to direct a microscope's field of view to the brain for imaging of neuronal activity. Such tracking microscopes have been developed in recent years, and depending on the implementations, they can image the whole nervous system of the targeted animal with cellular resolution. A Comment by Calarco and Samuel highlights the developments in this area and points out the challenges associated with the analysis of the large datasets acquired in such studies.
While we chose imaging in freely behaving animals as Method of the Year 2018 , it is important to keep in mind that behavioral studies are not limited to the imaging of neuronal activity. Such studies also need to monitor behavior, in order to correlate behavior and neuronal activity, and may also manipulate neuronal activity to determine causality in neuronal circuitry. Researchers typically monitor behavior by capturing videos of the animals' actions. In this issue, an Article by Pereira et al. describes a computational tool called LEAP for conducting detailed behavioral studies by tracking body parts in flies and mice. The researchers applied LEAP to determine animal pose and gait. In another Article, Lee et al. describe a compressed sensing strategy used with Caenorhabditis elegans to identify the neurons controlling the speed of locomotion. By inhibiting neuronal activity in overlapping sets of neurons and using behavioral data, the researchers identified the key neurons for controlling speed from fewer measurements than anticipated.

Much progress has been made toward achieving a mechanistic understanding of circuits and behaviors in head-fixed or otherwise restrained animals. Now the challenge will be to devise and improve computational approaches for efficient analysis of the large datasets being generated. But with the tools in place to image brain activity in freely behaving animals and the possibility of combining these tools with sophisticated behavioral analyses and neuronal manipulations, exciting progress in studying more complex behaviors can be expected over the next few years.

We wish our authors, reviewers and readers a happy 2019 !

Published online: 20 December 2018 https://doi.org/10.1038/s41592-018-0292-8 